\title{
Successful Treatment of Refractory Autoimmune Hemolytic Anemia (AIHA) in a Child, Based on Iranian Traditional Medicine: A Case Report
}

\author{
Fatemeh Jafari ${ }^{1}(\mathbb{D})$, Gholamreza Kordafshari ${ }^{1}(\mathbb{D})$, Nahid Norouzi-Gheidari ${ }^{2}$ (D), \\ Mohammad Hossein Ayati ${ }^{1,3 *}$ (D)
}

1. Dept. of Traditional Medicine, School of Traditional Medicine, Tehran University of Medical Sciences, Tehran, Iran

2. School of Physical and Occupational Therapy, Faculty of Medicine, McGill University, Montreal, QC, Canada

3. Dept. of Medical History, School of Traditional Medicine, Tehran University of Medical Sciences, Tehran, Iran

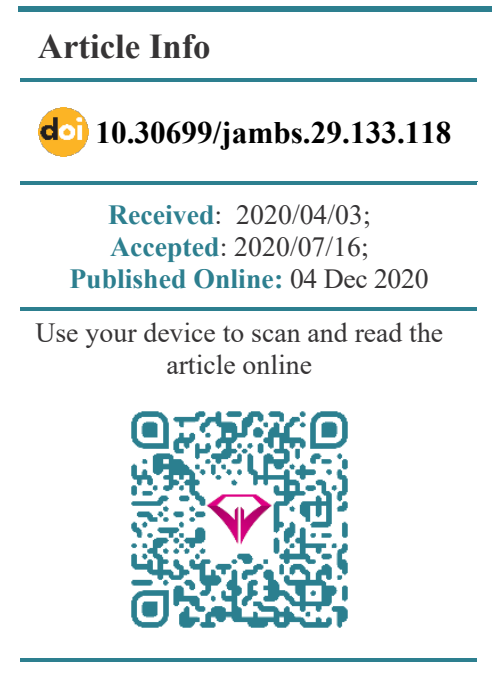

Corresponding Information Mohammad Hossein Ayati, Dept. of Traditional Medicine, School of Traditional Medicine, Tehran University of Medical Sciences, Tehran, Iran.

E-Mail:

mh-ayati@tums.ac.ir

\section{ABSTRACT}

\begin{abstract}
Autoimmune hemolytic anemia (AIHA) is a heterogeneous and relatively unknown disease caused by premature immune destruction of red blood cells. While its occurrence is uncommon among children, it is sometimes severe and resistant to treatment. The warm-reactive type contains $70 \%$ to $80 \%$ of all cases, in which the first-line treatment is considered to be a steroid. Moreover, splenectomy, rituximab (a monoclonal antibody), and immunosuppressive drugs are used in refractory cases, with unclear efficacy and deep suppression of the immune system, which consequently lead to various side effects. This study reports the successful treatment of a life-threatening case using a new method. In this regard, it was stated that using the capacity of Iranian traditional medicine (ITM) as one of the complementary therapies can help in the treatment of this disease.
\end{abstract}

In this case report, we documented the successful treatment of a severe and refractory warm AIHA in a boy, who was resistant to the currently recommended treatments such as corticosteroids, rituximab, and cyclosporine at different time periods. Based on ITM, a novel treatment was performed, which was daily swallowing 4-6 live small freshwater fishes (from Cyprinidae family) for an eight-week period and later being tapered. As a result, this treatment had a rapid response with no side effects. At the time of performing this study, the patient was in his 5th-year diseasefree period. For future research, we recommend the researchers to study the use of this novel treatment in case of resistance to the current established therapies of warm AIHA disease.

Keywords: Alternative medicine, Autoimmune hemolytic anemia, Complementary therapies, Traditional medicine

\section{Introduction}

Autoimmune hemolytic anemia (AIHA) is a relatively unknown and heterogeneous disease with an incidence of $1-3$ cases per 100,000/year and a mortality rate of approximately $11 \%$ (1). Hemolysis is resulted from autoantibodies binding to the red blood cell (RBC) membrane antigens, which lead to their premature destruction by macrophages of the reticuloendothelial system (2). Accordingly, half of the cases are uncanny and primary, and the rest of them are caused by an underlying condition including malignancy, infection, immune disease, and medication. The presence of macrocytic normocytic anemia and reticulocytosis, increased indirect bilirubin, increased serum lactate dehydrogenase, decreased serum haptoglobin, and blood smear with polychromasia or more specific features such as spherocytes or agglutination confirm the existence of hemolytic anemia (3). Also, the diagnosis is performed based on the result of the direct antiglobulin test (DAT). Moreover, the classification is based on the DAT pattern and the maximum temperature response of the autoantibodies to the RBC. Notably, $70 \%$ to $80 \%$ of all cases are classified as the warm type. On the other hand, the cold type includes $15 \%$ to $20 \%$ of all cases and the mixed type of AIHA includes of $10 \%$ of them (4).

Treatment depends on the type and severity of AIHA as well as the underlying disease. In warm AIHA, the first-line treatment is a steroid and the second-line is splenectomy or Rituximab. Moreover, in the presence of anemia and clinical findings on the decreased tissue perfusion such as confusion, emergency pRBC transfusion is required (5). Rituximab (which is a monoclonal antibody anti CD20) has been effectively 
used instead of splenectomy in treating AIHA in both warm and cold types (6). Notably, rituximab has been recently studied as a first-line co-administration with steroid, which appears to produce a longer response compared to steroid alone (7). In recurrence cases, azathioprine, cyclosporine, and mycophenolate mofetil are considered as the third-line therapies. Also, in refractory cases, therapeutic options are as follows: Low dose prednisolone, cyclophosphamide, Danazol, and Bortezomib (a monoclonal antibody). Notably, hematopoietic stem cell transplantation is limited to single cases in refractory cases (8).

This study aimed to state that, using the capacity of Iranian traditional medicine (ITM), as one of the complementary therapies, can be considered helpful in diseases treatment. In this case report, we documented our case using a new method based on ITM, which led to the successful treatment of a severe and refractory warm AIHA in a boy who was resistant to the currently recommended treatments using corticosteroids, rituximab, and cyclosporine at different time periods. It was shown that this treatment can have a rapid response time with no side effects. At the time of performing this study, the patient was in his 5th year of disease-free period.

\section{Case Presentation}

An 11-year-old male child was referred to the Children's Medical Center of Tehran University of Medical Sciences. Notably, in his medical history, there was weakness, lethargy, and pallor from the past three weeks, following three days of diarrhea. He had no family history of hematological problems or any autoimmune disease. He was born and raised in Tehran city. At the time of referral, his height and weight were $155 \mathrm{~cm}$ and $50 \mathrm{~kg}$, respectively.

\section{Clinical Findings and Diagnostic Assessment}

Jaundice and pallor were noted on the physical exam and his heart rate was 115 beats per minute. Laboratory test results showed hemoglobin level as $7.9 \mathrm{~g} / \mathrm{dl}$, mean corpuscular volume as 110.2 FL, white blood cells of $8.71 \times 10^{9} / 1$, platelets as $227 \times 10^{9} / 1$, total bilirubin of $5.1 \mathrm{mg} / \mathrm{dl}$, direct bilirubin as $0.4 \mathrm{mg} / \mathrm{dl}$, and serum lactate dehydrogenase as $1760 \mathrm{u} / \mathrm{l}$. Moreover, the peripheral blood smear showed polychromasia, spherocytes, anisocytosis, and NRBC (2\%). The urine analysis test result was negative for hemoglobin and myoglobin, and also positive for granular casts. In addition, the liver and kidney function tests were normal. DAT was positive for IgG autoantibodies and negative for IgM autoantibodies and complement fixation. Antinuclear antibody and anti-deoxyribonucleic acid levels were negative in the serum. Furthermore, serum levels of the complement components including $\mathrm{C} 3, \mathrm{C} 4$, and $\mathrm{CH} 50$ were normal. According to the laboratory findings, the patient was diagnosed with a warm type of AIHA, besides the symptoms and clinical findings.

\section{Therapeutic Intervention}

\section{Modern Medicine Intervention}

Oral prednisone at a dose of $2 \mathrm{mg} / \mathrm{kg} /$ day was started at the first admission point (July 16, 2014) and lasted for 4 weeks, followed by a slow taper for a 3-month period. Relapse occurred by passing 7 weeks from the first treatment. At this time, cyclosporin was administered at a dose of $2 \mathrm{mg} / \mathrm{kg} /$ day with no hematological responses, but with the side effects of facial edema and hairy back. For this reason, cyclosporine was discontinued after 3 months of usage. At that time, rituximab was weekly administered at a dose of $375 \mathrm{mg} / \mathrm{m}^{2}$ for a 4-week period. Accordingly, no response was observed 2 months after the first administration. The pRBC transfusion was also administered alternately from the 13th to the 30th weeks to avoid a cardiovascular compromise due to severe anemia (Figure 1). The frequency of blood transfusion was $1 \mathrm{U}$ red blood cells at each time. IVIg transfusion was also performed at the 13th week. Figure 1 shows the therapeutic agents and their clinical responses to hemoglobin over time.

\section{ITM Intervention}

Despite the above-mentioned treatments, the symptoms of the disease worsened. The patient was then taken to the Traditional Medicine Clinic at Tehran University of medical sciences. He was suddenly afraid of everything three weeks before the disease onset. At the time of admission, he was weak, pale, and yellowish with palpitations. The Laboratory findings at this time were as follows: hemoglobin 8/05 g/dl, WBC $8 / 45 \times 10^{9} / 1$, PLT $379 \times 10^{9} / 1$, Total bilirubin $10 / 19 \mathrm{~g} / \mathrm{dl}$, and direct bilirubin $0.52 \mathrm{~g} / \mathrm{dl}$. The peripheral blood smear contained atypical cells (spherocytosis 2+, polychromasia $1+$, NRBC 4\%, and Band cell 1\%) and DAT was positive.

A special treatment based on ITM was prescribed for the patient. Correspondingly, this treatment consisted of swallowing the live small freshwater fish (family Cyprinidae). The live small fish was placed in a cup of water, and then water and fish were swallowed together. It was hard at first, but then he got used to it. Four to six live small fishes were swallowed daily in divided doses in the morning and evening for a duration of about two months, which were then reduced over a month gradually. After the start of this treatment, the hemoglobin levels increased in a short time and reached over $12 \mathrm{~g} / \mathrm{dl}$ during 3 weeks. At this point, although the DAT was positive, the PBS had no atypical cells. Three months after the treatment, laboratory results were as follows: hemoglobin 16.1 $\mathrm{g} / \mathrm{dl}$, WBC $6.10 \times 10^{9} / 1$, platelets $188 \times 10^{9} / 1$, total bilirubin $1.4 \mathrm{~g} / \mathrm{dl}$, and direct bilirubin $0.3 \mathrm{~g} / \mathrm{d}$. DAT have also become negative. At this point, the treatment was stopped. Notably, the effect of this treatment was quick and surprising, and no side effects were observed. 


\section{Follow-up and Outcomes}

The boy has remained disease-free 5 years after the first presentation and the hemoglobin level has remained within the normal range.

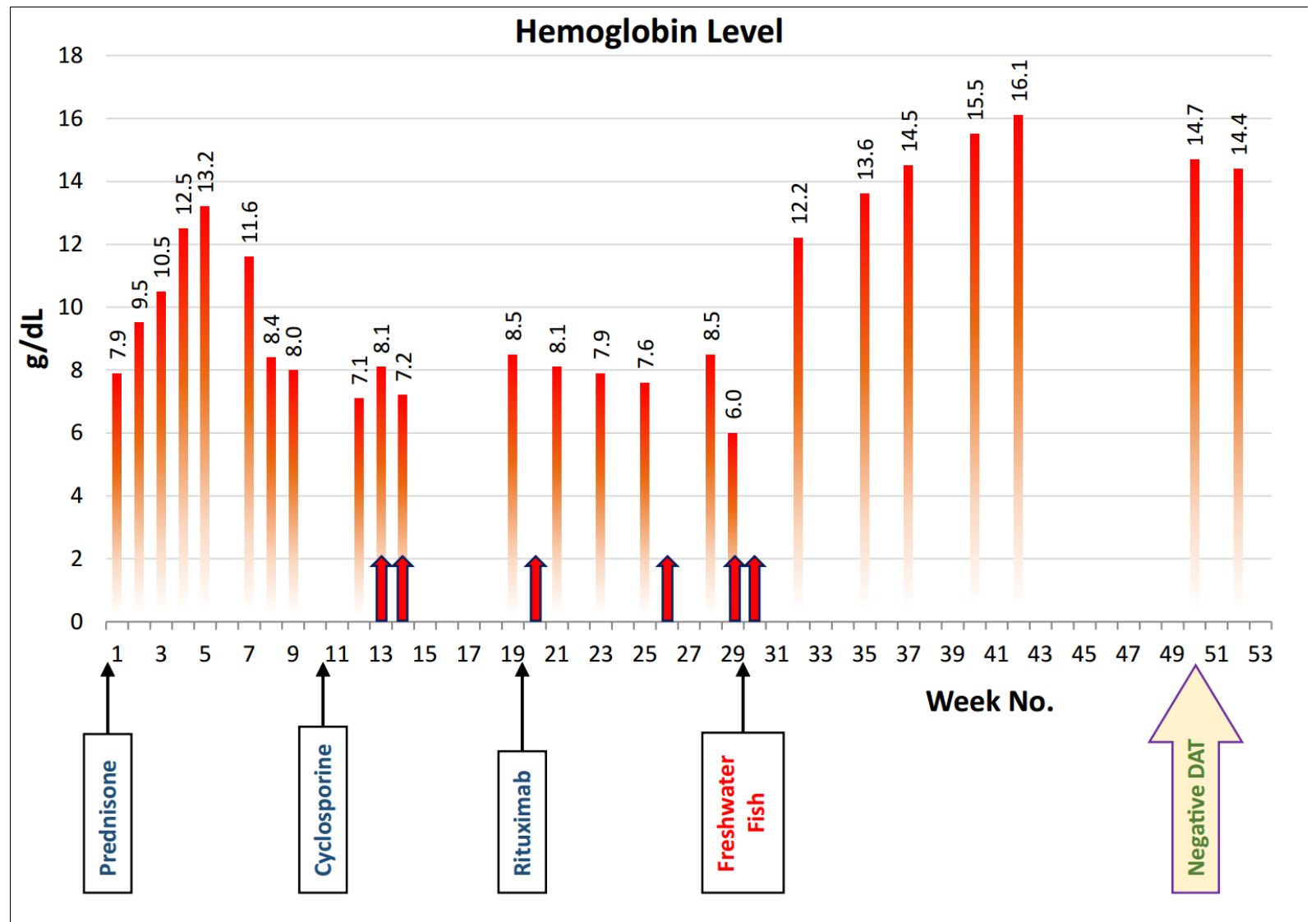

Figure 1. Hemoglobin over time

\section{Discussion}

The pathophysiology of autoantibodies production in AIHA is unknown yet, but it probably involves an immune dysregulation as well as abnormal autoantigen responses (7). Due to having difficulty in performing randomized controlled trials, the treatment and prognosis of AIHA are still major challenges (9). Immunosuppressants used for severe anemia or mixed serology have high rates of mortality and morbidity (10). It was found that rituximab can lead to secondary hypogammaglobulinemia, prolonged B-cell lymphopenia, and recurrent or severe infections (11). Also, some targeted therapies have recently been explored as follows: CD52 (alemtuzumab), B cell receptors and proteasome inhibitors (ibrutinib, bortezomib), complementary inhibitors (eculizumab), and targeting $\mathrm{T}$ cells (belimumab). Bone marrow biopsy can provide several clues to targeted therapies and new immunomodulatory drugs. Although AIHA is considered as benign, chronic/refractory cases are challenging even for experts of this field (12).
In this study, we reported a successful ITM-based treatment leading to some surprising results in severe refractory cases of warm AIHA in a boy. In this special treatment, 4-6 small live fishes were daily swallowed. By passing 3 weeks from the start of treatment, the hemoglobin levels reached over $12 \mathrm{~g} / \mathrm{dl}$ and the clinical symptoms have also improved. After 3 months, hemoglobin levels reached over $16 \mathrm{~g} / \mathrm{dl}$ and other laboratory results were within the normal range. Notably, DAT was also negative. At the start of this treatment, the patient had no longer received treatment from modern medicine. Our observation is special because the effect of the treatment is quick and complete with no complications.

It is a new treatment and how the drug regulates the immune system and prevents the destruction of red blood cells leading to anemia, are still unknown, which need to be studied in future. According to the principles of ITM, in this disease, the liver has a lot of heats by excessive activity causing the destruction of red blood 
cells, which finally leads to jaundice $(13,14)$. Fish has a cold temperament, so its consumption reduces the heat of the liver and prevents its overactivity. The production of bile also decreases, which improves jaundice. The consumption of fish in jaundice is recommended by swallowing live small fish in sources of ITM. The amount is up to seven per each day with an empty stomach (15). It should be noted that using this treatment for jaundice in Iranian's folk medicine has been heard sometimes in the language of the elderly. However, the observation of the dramatic response to novel treatment in this patient indicated a potential critical role of ITM in this unique setting. The existence of a disease-free period for five years makes this case report more important.

\section{Conclusion}

The current novel therapeutic approaches are known as a necessity because of the severity and lifethreatening nature of AIHA. ITM is one of the complementary and alternative medicines providing different therapeutic methods for diseases, which can be combined with conventional drug therapies. The new treatment options need a long way to go before being recommended as effective and safe treatments. At least, the use of such treatment options (swallowing freshwater fishes) in refractory AIHA cases can be suggested to reduce morbidity and mortality of the disease.

\section{Informed Consent}

Written permission for publication of this case study was obtained from the patient's parents.

\section{Ethical considerations}

Ethical issues (Including plagiarism, informed consent, misconduct, data fabrication and/or falsification, double publication and/or submission, redundancy, etc.) have been completely observed by the authors.

\section{Funding and support}

This research resulted from an independent research without receiving any financial support.

\section{Conflict of Interest}

Authors declared no conflict of interest.

\section{References}

1. Park SH. Diagnosis and treatment of autoimmune hemolytic anemia: classic approach and recent advances. Blood Res. 2016;51(2):69-71. (DOI:10.5045/br.2016.51.2.69)

2. Liebman HA, Weitz IC. Autoimmune hemolytic anemia. Med Clin North Am. 2017;101(2):351-9 (DOI:10.1016/j.mcna.2016.09.007)

3. Hill A, Hill QA. Autoimmune hemolytic anemia. Hematology. 2018;2018(1):382-9. (DOI:10.1182/asheducation-2018.1.382)

4. Chaudhary RK, Das SS. Autoimmune hemolytic anemia: From lab to bedside. Asian J Transfus Sci. 2014;8(1):5-12 (DOI:10.4103/0973-6247.126681)

5. Naik R. Warm autoimmune hemolytic anemia. Hematol Oncol Clin North Am. 2015;29:445-53. (DOI:10.1016/j.hoc.2015.01.001)

6. Reynaud Q, Durieu I, Dutertre M, et al. Efficacy and safety of rituximab in auto-immune hemolytic anemia: A meta-analysis of 21 studies. Autoimmun Rev. 2015;14:304-13. (DOI:10.1016/j.autrev.2014.11.014)

7. Dierickx D, Kentos A, Delannoy A. The role of rituximab in adults with warm antibody autoimmune hemolytic anemia. J Am Soc Hematol. 2015;125(21):3223-9. (DOI:10.1182/blood-201501-588392)

8. Jäger U, Barcellini W, Broome CM. Diagnosis and treatment of autoimmune hemolytic anemia in adults: Recommendations from the First International Consensus Meeting. Blood Rev. 2020;41:100648. (DOI:10.1016/j.blre.2019.100648)

9. Go RS, Winters JL, Kay NE. How I treat autoimmune hemolytic anemia. Blood. 2017;129:2971-9. (DOI:10.1182/blood-2016-11693689)

10. Makis A, Kanta Z, Kalogeropoulos D, Chaliasos N. Anti-CD20 treatment of autoimmune hemolytic anemia refractory to corticosteroids and azathioprine: A pediatric case report and mini review. Case Rep Hematol. 2018;2018:8471073. (DOI:10.1155/2018/8471073)

11. Ducassou S, Leverger G, Fernandes H, et al. Benefits of rituximab as a second-line treatment for autoimmune haemolytic anaemia in children: a prospective French cohort study. $\mathrm{Br} J$ Haematol.2017; 177:751-8. (DOI:10.1111/bjh.14627)

12. Barcellini W, Fattizzo B, Zaninoni A. Current and emerging treatment options for autoimmune hemolytic anemia. Expert Rev Clin Immunol. 2018;14(10):857-72.

(DOI:10.1080/1744666X.2018.1521722) 
13. Azam Khan M. Exir Azam (Persian) Volume 1. Tehran: Institute of Meical History, Islamic Medicine and Complementary Medicine; 2008.

14. Ibn-e-Sina AH. Al-qanun fit-tib (The canon of medicine). Beirut, Lebanon: Alaalami Beirut lib Press; 2005.
15. Ibn al-Nafis. Ash-shamel fit-teb. Beirut: Dar Alkotob Al-ilmiyah Publication; 2004.

\section{How to Cite This Article:}

Jafari F, Kordafshari G, Norouzi Gheidari N, Ayati M H. Successful Treatment of Refractory Autoimmune Hemolytic Anemia (AIHA) in a Child, Based on Iranian Traditional Medicine: A Case Report. J Adv Med Biomed Res. 2021; 29 (133) :118-122

\section{Download citation:}

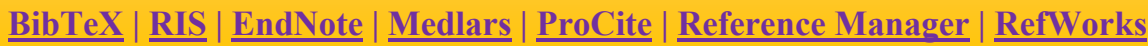

\section{Send citation to:}

Mendeley 2 Zotero (i) RefWorks $\underline{\text { RefWorks }}$ 